\title{
Detection of cancer cells and gene expression of cytokines in the peritoneal cavity in patients with gastric cancer
}

\author{
Youji Fukumoto, Masahide Ikeguchi, Sachiko Matsumoto, Masashi Inoue, Tomohiro Osaki, Kenji Fukuda, \\ Hiroaki Saito, Shigeru Tatebe, and Shun-Ichi Tsujitani \\ Department of Surgery, Division of Surgical Oncology, Faculty of Medicine, National University Corporation Tottori University, \\ 36-1 Nishi-cho, Yonago 683-8504, Japan
}

\begin{abstract}
Background. The gene expression of the cytokines interleukin-2 $(I L-2)$ and $I L-10$ in peritoneal washings was examined in relation to the presence of cancer cells in the peritoneal cavity in patients with gastric cancer.

Methods. Total RNA was extracted from $50-\mathrm{ml}$ peritoneal wash samples from 124 patients (gastric cancer, $n=110$; controls, $n=14)$. Carcinoembrionic antigen (CEA) messenger RNA (mRNA) was used to identify the number of cancer cells in peritoneal wash samples by a real-time reverse transcription-polymerase chain reaction (RT-PCR) method, which method was also used to assay the $I L-2$ and $I L-10$ gene expression levels.

Results. In the 14 control samples, CEA mRNA was not detected, while CEA mRNA was detected in 2 of the 51 stage I gastric cancer patients. Thus, the specificity of this method for the detection of cancer cells in peritoneal wash samples was 97\% (63/65). The CEA-based real-time RT-PCR method demonstrated greater prognostic impact than the traditional cytological method. $I L-2$ gene expression in peritoneal wash samples that were CEA mRNA-positive was suppressed compared with that in peritoneal wash samples that were CEA mRNA-negative, while $I L-10$ gene expression did not differ according to the CEA mRNA findings.

Conclusion. The detection of small numbers of cancer cells in peritoneal wash samples from patients with advanced gastric cancer is a good marker for peritoneal metastatic recurrence. In the peritoneal cavity, cancer cells may escape from immune surveillance by controlling the expression of cytokines.
\end{abstract}

Key words Cytokine - Gastric cancer - Intraperitoneal free cancer cells - Real-time reverse transcriptase polymerase chain reaction

Offprint requests to: $\mathrm{M}$. Ikeguchi

Received: January 23, 2006 / Accepted: June 7, 2006

\section{Introduction}

In advanced gastric cancer, peritoneal metastasis or intraperitoneal free cancer cells are important factors for a poor prognosis [1-3]. Peritoneal washing cytology has been introduced for the detection of cancer cells in the peritoneal cavity in patients with gastric cancer [4]. However, with this method, it is difficult to identify small numbers of cancer cells in peritoneal washings. Recent advances in the real-time reverse transcriptionpolymerase chain reaction (RT-PCR) method have enabled us to identify small numbers of cancer cells in the peritoneal cavity [5]. In the present study, we investigated the clinical importance of the detection of small numbers of cancer cells in peritoneal wash samples from gastric cancer patients.

The peritoneal cavity is thought to be a compartment in which immunologic host-tumor interaction can occur. Recent studies have demonstrated that the cytokine network plays important roles in the immunosuppressive and immunostimulatory properties of cancerrelated ascites fluid [6,7]. To clarify the relationships between the gene expression levels of cytokines and the detection of cancer cells in the peritoneal cavity, we examined the gene expression levels of interleukin-2 (IL-2) and $I L-10$ in peritoneal wash samples obtained from gastric cancer patients.

\section{Methods}

\section{Sample collection}

A total of 110 gastric cancer patients (72 men and 38 women; mean age, 66 years; range, 26-88 years) and 14 patients with cholecystolithiasis (controls, 6 men and 8 women; mean age, 50 years; range, 14-83 years) operated between January 2002 and March 2005 were enrolled. In the 110 patients with primary gastric cancer 
(stage I, $n=51$; II, $n=14$; III, $n=20$; and IV, $n=25$ ), gastrectomy was performed in 96 patients; gastrectomy was not performed in the remaining 14 patients because of peritoneal dissemination (12 cases) or extended lymph node metastasis ( 2 cases). Aliquots of $150 \mathrm{ml}$ of saline were introduced into the Douglas cavity at the beginning of the operation and aspirated soon after gentle stirring. Then $50-\mathrm{ml}$ the peritoneal washings was submitted to cytological diagnosis and the remaining $50 \mathrm{ml}$ was prepared for RNA extraction. The present study conformed to the ethical standards of the World Medical Association Declaration of Helsinki. Tumor stage and pathological classification were described according to the Japanese Classification of Gastric Carcinoma [8].

\section{Cytological diagnosis of intraperitoneal free cancer cells}

The collected saline peritoneal wash sample was centrifuged for $5 \mathrm{~min}$ at $2000 \mathrm{~g}$. The cell pellet was smeared onto four slides, two for Papanicolaou staining and two for Giemsa staining. These specimens were examined at the pathology laboratory at our hospital by physicians licensed by the Japanese Society of Clinical Cytology.

\section{Human gastric cancer cell lines}

Human gastric cancer cell lines (MKN-28, MKN-45, MKN-74, and KATO-III) were maintained in RPMI-1640 medium (Cosmo Bio, Tokyo, Japan) supplemented with $10 \%$ fetal calf serum (Cansera International, Ontario, Canada) in a humidified atmosphere containing $5 \% \mathrm{CO} 2$ at $37^{\circ} \mathrm{C}$.

\section{Real-time RT-PCR assay}

The peritoneal washings were centrifuged for $10 \mathrm{~min}$ at $400 \mathrm{~g}$. Total RNA from the pellets of the peritoneal washings was isolated using RNeasy Mini Kits (Qiagen, Hilden, Germany) according to the manufacturer's protocol. Complementary DNA (cDNA) was synthesized with $1 \mu \mathrm{g}$ of total RNA with Ready-to-Go You-Prime First-Strand Beads (Amersham Pharmacia Biotech, Piscataway, NJ, USA) and the final volume of cDNA solution was adjusted to $33 \mu \mathrm{l}$ with diethylpyrocarbonate (DEPC)-treated water. Primers and TaqMan (Perkin-Elmer Applied Biosystems, Foster City, CA, USA) probes for CEA were as follows: forward primer, 5'-GGCCACTGTCGGCATCAT-3'; reverse primer, 5'-GGAAGAAGCAAAACAACTGTCAGTC-3'; and probe, $5^{\prime}$-CAGCCCTGGTGTAGTTTCTTCATT TCAGGA-3'. Primers and TaqMan probes for TATA box-binding protein (TBP; as the endogenous RNA control), IL-2, and IL-10 were obtained from Perkin-
Elmer Applied Biosystems. The PCR solution (50 $\mu \mathrm{l})$ was composed of $1 \mu \mathrm{l}$ of cDNA solution, $5 \mathrm{pmol}$ of the forward and reverse primers, $10 \mathrm{pmol}$ of internal probe, and TaqMan Universal PCR Master Mix (Perkin-Elmer Applied Biosystems). PCR was carried out after incubation at $50^{\circ} \mathrm{C}$ for $2 \mathrm{~min}$ and denaturing at $95^{\circ} \mathrm{C}$ for $10 \mathrm{~min}, 45$ cycles at $95^{\circ} \mathrm{C}$ for $15 \mathrm{~s}$, and $61^{\circ} \mathrm{C}$ for $1 \mathrm{~min}$. Quantification of gene expression was performed by a real-time quantitative RT-PCR (Gene Amp 5700 Sequence Detection System [Perkin-Elmer Applied Biosystems]) [9].

\section{Relative numbers of cancer cells in the peritoneal wash samples}

Different concentrations of KATO-III cells (10 to $\left.10^{6}\right)$ were added to $1.5-\mathrm{ml}$ peripheral blood $(\mathrm{PB})$ samples from one healthy volunteer $\left(6 \times 10^{6}\right.$ peripheral blood granulocytes). CEA mRNA expression levels from different concentrations of KATO-III were identified by the real-time RT-PCR. The experiments were repeated three times. When the plots represented the log of KATO-III cells in $6 \times 10^{6}$ granulocytes on the $x$ axis and cycle numbers on the $y$ axis, an equation was derived from the line of the CEA calibration curve [10]. The formula for CEA was $y=40-3 x\left(r^{2}=0.994\right)$. For each of the experimental samples, the amounts of CEA mRNA were determined from this standard curve. TBP transcripts were monitored as a control to quantify the transcripts.

\section{Quantification of cytokine gene expression}

The method for quantification of cytokine gene expression was described previously [11]. Briefly, for each reaction tube, the fluorescence signal of the reporter dye was divided by the fluorescence signal of the passive reference dye to obtain the normalized reporter signal $(\mathrm{Rn})$. The threshold line was set at $\mathrm{Rn}=0.1$. The point at which the amplification plot crossed this threshold was defined as $\mathrm{Ct}$, which represented the cycle number at this point. Each sample was normalized by the gene expression of TBP. TBP was chosen because there are no known retropseudogenes [12], and the gene expression level of $T B P$ is lower than those of $I L-2$ and $I L-10$. The cDNAs were prepared from 1.5-ml PB samples from eight healthy volunteers [9]. The $\Delta \mathrm{Ct}$ values for $I L-2$ and $I L-10$ in the PB samples were determined by subtracting the $I L-2$ and $I L-10 \mathrm{Ct}$ values from the TBP $\mathrm{Ct}$ value. These mean $\Delta \mathrm{Ct}$ values for $I L-2$ and $I L-10$ in the PB samples were used as calibrators (2.0 and 1.0, respectively). In each sample, the expression levels of the target genes were obtained by the following formulas, $\Delta \mathrm{Ct} I L-2$ (sample) $/ \Delta \mathrm{Ct} I L-2$ (calibrator) and $\Delta \mathrm{Ct}$ $I L-10$ (sample) $/ \Delta \mathrm{Ct} I L-10$ (calibrator). Since $\Delta \mathrm{Ct} I L-2$ 
(calibrator) was 2.0 and $\Delta \mathrm{Ct} I L-10$ (calibrator) was 1.0 , the relative expression levels of the $I L-2$ gene and $I L-10$ gene in this sample were $\Delta \mathrm{Ct} I L-2$ (sample)/2.0 and $\Delta \mathrm{Ct}$ IL-10 (sample)/1.0.

\section{Statistical analysis}

The $\chi^{2}$ test and Fisher's exact probability test were used to compare the distribution of individual variables among the patient groups. Differences in numerical data between two groups were evaluated using the Mann-Whitney $U$-test, and the Kruskal-Wallis test was used for evaluating differences among three or more groups. Survival rates were calculated using the KaplanMeier method. The log rank test was used for comparisons of two survival curves. A $P$ value of less than 0.05 was considered statistically significant.

\section{Results}

\section{Sensitivity and reliability of $C E A m R N A$ probes}

CEA mRNA expression was detected in all four cell lines (MKN-28, MKN-45, MKN-74, and KATO-III). The CEA mRNA probe can detect 10 cancer cells in 6 $\times 10^{6}$ granulocytes. We found no CEA mRNA expression in the peritoneal wash samples taken from the 14 noncancerous patients. However, we found CEA mRNA expression in the peritoneal wash samples from 2 of the 51 stage I gastric cancer patients. One was a 60year-old man and the disease was classified as stage IA. He had $42 \mathrm{CEA}$-positive cells $/ 6 \times 10^{6}$ granulocytes in the peritoneal wash sample and was followed for 33 months without recurrence. The other patient was a 76-years- old man whose disease was classified as stage IB. He had 19 CEA-positive cells $/ 6 \times 10^{6}$ granulocytes in the peritoneal wash sample and was followed for 34 months without recurrence. So, we decided that the results for these two stage I patients were false-positives. From our data, the specificity of CEA for the detection of small numbers of cancer cells in peritoneal wash samples was 97\% (63/65) and CEA mRNA was thought to be a sensitive and reliable probe to identify small numbers of cancer cells.

\section{Peritoneal metastasis and cytological findings in peritoneal washings}

Macroscopic peritoneal metastasis $(\mathrm{P})$ was detected in 16 patients and intraperitoneal free cancer cells recognized by cytology (CY) were detected in 16 patients. We divided the 110 gastric cancer patients into four subgroups according to $\mathrm{P}$ and $\mathrm{CY}$ status. Eighty-eight patients were $\mathrm{CY}(-) / \mathrm{P}(-), 6$ were $\mathrm{CY}(-) / \mathrm{P}(+), 6$ were $\mathrm{CY}(+) / \mathrm{P}(-)$, and 10 were $\mathrm{CY}(+) / \mathrm{P}(+)$.

\section{$C E A m R N A$ expression in peritoneal washing from gastric cancer patients}

CEA mRNA was detected in the peritoneal wash samples from 33 gastric cancer patients (range, $4-1.5 \times$ $10^{6} / 6 \times 10^{6}$ granulocytes $)$. When we used the cutoff value (10 cancer cells $/ 6 \times 10^{6}$ granulocytes) used in a previous report [5], CEA mRNA was detected in the peritoneal wash samples of patients $27(24.5 \%)$. Figure 1 shows the relative numbers of cancer cells in the peritoneal wash samples of the 110 gastric cancer patients. Positivity for CEA mRNA in the peritoneal wash samples was as follows: $7 / 88(8 \%)$ with $\mathrm{CY}(-) / \mathrm{P}(-), 4 / 6$ (67\%) with

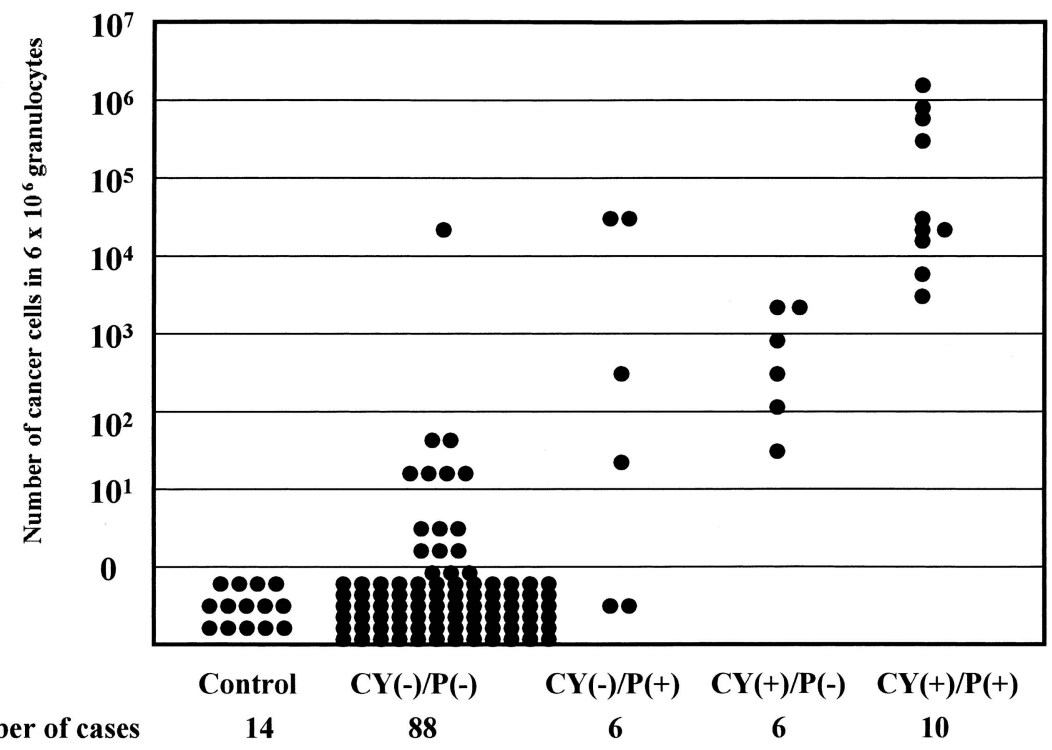

Fig. 1. Relative numbers of cancer cells in the peritoneal wash samples of 110 gastric cancer patients $C Y$, peritoneal free cancer cells detected by cytology; $P$, macroscopic peritoneal metastasis 
Table 1. Detection of CEA mRNA expression in peritoneal wash samples from patients with gastric cancer

\begin{tabular}{lccc}
\hline & $n$ & CY $(+)$ & CEA mRNA (+) \\
\hline Stage & & & \\
I & 51 & 0 & $2(4 \%)$ \\
II & 14 & 0 & 0 \\
III & 20 & 0 & $4(20 \%)$ \\
IV & 25 & 16 & $21(84 \%)$ \\
\hline
\end{tabular}

CY, peritoneal free cancer cells recognized by cytology

$\mathrm{CY}(-) / \mathrm{P}(+), 6 / 6(100 \%)$ with $\mathrm{CY}(+) / \mathrm{P}(-)$, and $10 / 10$ $(100 \%)$ with $\mathrm{CY}(+) / \mathrm{P}(+)$. CEA mRNA was detected in all 16 patients with $\mathrm{CY}(+)$. However, in 2 of the 6 patients with $\mathrm{CY}(-) / \mathrm{P}(+)$, we failed to find cancer cells in the peritoneal cavity by real-time RT-PCR. The correlation between the detection of CEA mRNA and tumor stage is indicated in Table 1.

\section{Correlation between detection of cancer cells in} peritoneal washings and cytokine gene expression

$I L-2$ gene expression was detected in $11 / 14$ (79\%) of the control patients and in 85/110 (77\%) of the gastric cancer patients, and $I L-10$ gene expression was detected in all of the control and in 108/110 (98\%) of the gastric cancer patients. In the 110 gastric cancer patients, the mean $I L-2$ gene expression level in the 27 patients who were CEA-positive (1.1; range, 0-3.6) was lower than that in the 83 CEA-negative patients (1.8; range, $0-5.8$; $P=0.044)$. On the other hand, the mean $I L-10$ gene expression level in the 27 CEA-positive patients (8.2; range, 3-15) was not different from that in the 83 CEAnegative patients $(8.7$; range, $0-17.8 ; P=0.466)$.

\section{Correlation between detection of cancer cells in peritoneal washings and patients' prognoses}

The overall 3-year survival rate of the 88 patients with $\mathrm{CY}(-) / \mathrm{P}(-)$ was $78 \%$; all 6 of the patients with $\mathrm{CY}(-) /$ $\mathrm{P}(+)$ died within 19 months after surgery (mean, 11.8 months); all 6 of the patients with $\mathrm{CY}(+) / \mathrm{P}(-)$ died within 9 months (mean, 6.8 months); and all 10 of the patients with $\mathrm{CY}(+) / \mathrm{P}(+)$ died within 14 months (mean, 6.7 months). No difference was observed in mean survival periods after surgery among the three groups, of $\mathrm{CY}(-) / \mathrm{P}(+), \mathrm{CY}(+) / \mathrm{P}(-)$, and $\mathrm{CY}(+) / \mathrm{P}(+)$. CEA mRNA was detected in 4 of the 20 patients with stage III disease, and all of these 20 patients underwent curative gastrectomy. The disease-free survival curves of the 16 CEA mRNA-negative patients and the 4 CEApositive patients are shown in Fig. 2. All 4 patients who were CEA mRNA-positive developed peritoneal metastasis and 3 of the 4 died within 18 months after opera-

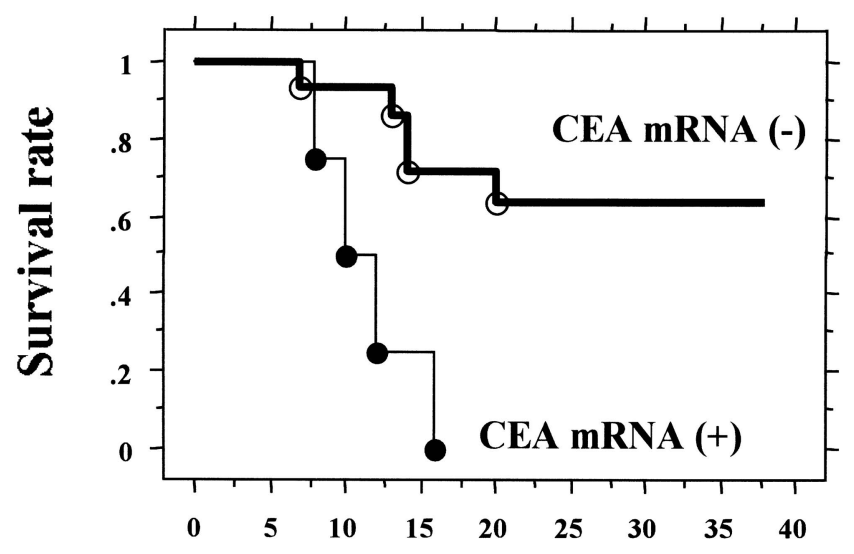

Time after surgery (months)

Fig. 2. Disease-free survival curves of 16 stage III gastric cancer patients who were carcinoembryonic antigen (CEA) mRNA-negative and 4 stage III patients who were CEAmRNA positive are shown. The difference was significant $(P=$ 0.002 )

tion. The remaining patient was alive with peritoneal metastasis in June 2005 (22 months after surgery). Five of the remaining 16 stage III patients developed recurrence foci, in the liver $(n=2)$, lymph node $(n=2)$, and peritoneum $(n=1)$.

\section{Discussion}

Recently, it has been shown that small numbers of cancer cells in peritoneal washings can be detected by using real-time RT-PCR [13]. For detecting intraperitoneal free cancer cells, a CEA mRNA probe is widely used, and real-time RT-PCR using a CEA mRNA probe is a more reliable method for identifying small numbers of cancer cells than cytology [4], but we need to pay careful attention when analyzing the results. The reliability of this marker for the detection of small numbers of cancer cells has been discussed. Kodera et al. [13] reported that the specificity of CEA in the detection of intraperitoneal free gastric cancer cells was $94 \%$. In the present study, we found that 2 of 65 cases were false-positives (0/14 in the controls and 2/51 in stage I gastric cancer patients). Thus from our data, the specificity of CEA in the detection of small numbers of cancer cells from peritoneal wash samples was $97 \%(63 / 65)$. Of our 20 stage III patients, 4 were CEA mRNA-positive. All 4 of these patients developed peritoneal metastasis within 18 months after operation. This result suggests that the use of real-time RT-PCR analysis of peritoneal wash samples is applicable for patients with advanced gastric cancer, because, CEA mRNA-based real-time RT-PCR is a strong prognostic marker for these patients. 
Six of our 16 patients with histologically confirmed peritoneal metastasis showed no free cancer cells by cytology, and we failed to find cancer cells in the peritoneal wash samples in 2 of these 6 patients even by the real-time RT-PCR method. These 2 patients underwent gastrectomy but died within 8 months due to peritoneal metastasis after operation. Peritoneal metastasis is widely recognized as a result of cancer-cell seeding from the primary tumor. However, another possibility is that, in some patients with peritoneal metastasis, lymphatic metastases to the peritoneal cavity may occur from metastatic lymph nodes or from the primary tumors. In such cases, peritoneal metastatic tumors may not face the peritoneal cavity directly and there may be no free cancer cells in the peritoneal cavity. Real-time RT-PCR is a useful method to detect small numbers of free cancer cells in the peritoneal cavity; however, not all patients with peritoneal metastasis may have free cancer cells in the peritoneal cavity.

Tumor-bearing hosts develop antitumor immune responses. In advanced gastric cancer, free cancer cells are frequently detected in the peritoneal cavity. Thus, the peritoneal cavity is a compartment in which immunologic host-tumor interaction can occur. IL-2 and IL-12 are produced by $\mathrm{T}$ cells and can affect many different cell types, such as lymphocytes, neutrophils, and macrophages. In particular, IL-2 activates natural killer (NK) cells, and CD4+ and CD8+ T cells (cytotoxic T lymphocytes), resulting in tumor destruction. Thus, IL2 and IL-12 have been used for intraperitoneal immunotherapy in patients with various cancers, including gastric cancer [14-16]. In the present study, we found that the gene expression of $I L-2$ was suppressed in the peritoneal cavity of patients who had free cancer cells in the peritoneal cavity. Peritoneal washings may contain not only immunocompetent cells but also tumor cells and normal mesothelial cells. So, this result suggests that cancer cells in the peritoneal cavity may suppress $I L-2$ gene expression in immunocompetent cells or normal mesothelial cells, and may change the environment of the peritoneal cavity so that it is suitable for tumor growth. Another possibility, that the low expression level of $I L-2$ in CEA-positive samples may correlate with a high cancer/T-cell ratio in the samples, can also be considered. On the other hand, Majima et al. [7] reported that the IL-10 protein level in peritoneal washings from patients with gastric cancer increased according to tumor progression. IL-10, a type-2 cytokine, inhibits type- 1 immune responses, and recent studies have reported that patients with carcinoma of the digestive tract more frequently showed type- 2 cytokines in the peripheral blood (PB) in comparison with normal subjects [17]. However, our results demonstrated that the gene expression level of $I L-10$ in peritoneal washings from patients who were CEA
mRNA-positive did not differ from that in the peritoneal washings from patients who were CEA mRNAnegative. So, the role of a type- 2 cytokine such as IL-10 in tumor progression in the peritoneal cavity in patients with advanced gastric cancer remains unclear.

Recently, a contrary finding on IL-2 function was reported, in which endogenous IL-2 promoted tumor cells growth and protected these cells from apoptosis [18]. Thus, the role of cytokines produced by cells in the peritoneal cavity in tumor growth in gastric cancer patients is still unclear. In future, the relationships between the gene and protein expressions of cytokines and tumor growth should be clarified.

\section{References}

1. Iitsuka Y, Shiota S, Matsui T, Murata Y, Kimura A, Koga S. Relationship between the cytologic characteristics of intraperitoneal free cancer cells and the prognosis in patients with gastric cancer. Acta Cytol 1990;34:437-42.

2. Ikeguchi M, Oka A, Tsujitani S, Maeta M, Kaibara N. Relationship between area of serosal invasion and intraperitoneal free cancer cells in patients with gastric cancer. Anticancer Res 1994; 14:2131-4.

3. Ribeiro U Jr, Safatle-Ribeiro AV, Zilberstein B, Mucerino D, Yagi OK, Bresciani CC, et al. Does the intraoperative peritoneal lavage cytology add prognostic information in patients with potentially curative gastric resection? J Gastrointest Surg 2006; 10:170-7.

4. Hayes N, Wayman J, Wadehra V, Scott DJ, Raimes SA, Griffin SM. Peritoneal cytology in the surgical evaluation of gastric carcinoma. Br J Cancer 1999;79:520-4.

5. Nakanishi H, Kodera Y, Yamamura Y, Ito S, Kato S, Ezaki T, et al. Rapid quantitative detection of carcinoembryonic antigen-expressing free tumor cells in the peritoneal cavity of gastric cancer patients with real-time RT-PCR on the lightcycler. Int J Cancer 2000;89:411-7.

6. Zeimet AG, Widschwendter M, Knabbe C, Fuchs D, Herold M, Müller-Holzner E, et al. Ascitic interleukin-12 is an independent prognostic factor in ovarian cancer. J Clin Oncol 1998;16:1861-8.

7. Majima T, Ichikura T, Seki S, Takayama E, Hiraide H, Mochizuki H. Interleukin-10 and interferon-gamma levels within the peritoneal cavity of patients with gastric cancer. J Surg Oncol 2001;78: 124-30.

8. Japanese Gastric Cancer Association. Japanese classification of gastric carcinoma - 2nd English edition. Gastric Cancer 1998;1: $10-24$.

9. Ikeguchi M, Ohro S, Maeda Y, Fukuda K, Yamaguchi K, Shirai $\mathrm{H}$, et al. Detection of cancer cells in the peripheral blood of gastric cancer patients. Int J Mol Med 2003;11:217-21.

10. Yajima T, Yagihashi A, Kameshima H, Kobayashi D, Furuya D, Hirata K, et al. Quantitative reverse transcription-PCR assay of the RNA component of human telomerase using the TaqMan fluorogenic detection system. Clin Chem 1998;44:2441-5.

11. Ikeguchi M, Matsumoto S, Murakami D, Kanaji S, Ohro S, Maeta $\mathrm{Y}$, et al. Gene expression levels of cytokines in peritoneal washings from patients with gastric cancer. Tumor Biol 2004;25: 117-21.

12. Linja MJ, Savinainen KJ, Saramaki OR, Tammela TL, Vessella RL, Visakorpi T. Amplification and overexpression of androgen receptor gene in hormone-refractory prostate cancer. Cancer Res 2001;61:3550-5.

13. Kodera Y, Nakanishi H, Ito S, Yamamura Y, Kanemitsu Y, Shimizu Y, et al. Quantitative detection of disseminated free cancer cells in peritoneal washes with real-time reverse 
transcriptase-polymerase chain reaction. A sensitive predictor of outcome for patients with gastric carcinoma. Ann Surg 2001;235: 499-506.

14. Lissoni P, Mandala M, Curigliano G, Ferretti G, Moro C, Ardizzoia A, et al. Progress report on the palliative therapy of 100 patients with neoplastic effusions by intracavitary low-dose interleukin-2. Oncology 2001;60:308-12.

15. Lenzi R, Rosenblum M, Verschraegen C, Kudelka AP, Kavanagh JJ, Hicks ME, et al. Phase I study of intraperitoneal recombinant human interleukin 12 in patients with Müllerian carcinoma, gastrointestinal primary malignancies, and mesothelioma. Clin Cancer Res 2002;8:3686-95.
16. Fu QG, Meng FD, Shen XD, Guo RX. Efficacy of intraperitoneal thermochemotherapy and immunotherapy in intraperitoneal recurrence after gastrointestinal cancer resection. World J Gastroenterol 2002;8:1019-22.

17. Tabata T, Hazama S, Yoshino S, Oka M. Th2 subset dominance among peripheral blood $\mathrm{T}$ lymphocytes in patients with digestive cancers. Am J Surg 1999;177:203-8.

18. Reichert TE, Kashii Y, Stanson J, Zeevi A, Whiteside TL. The role of endogenous interleukin-2 in proliferation of human carcinoma cell lines. Br J Cancer 1999;81:822-31. 